\title{
"Merolyn the Phone": A Study of Bluetooth Naming Practices (Nominated for the Best Paper Award)
}

\author{
Tim Kindberg $^{1}$ and Timothy Jones ${ }^{2}$ \\ ${ }^{1}$ Hewlett Packard Laboratories, \\ Filton Rd, Stoke Gifford, Bristol BS34 8QZ, UK \\ ${ }^{2}$ University of Central England, Birmingham, \\ School of Social Sciences, Perry Barr, Birmingham, B42 2SU, UK \\ timothy@hpl.hp.com, Timothy.Jones@uce.ac.uk
}

\begin{abstract}
This paper reports the results of an in-depth study of Bluetooth naming practices which took place in the UK in August 2006. There is a significant culture of giving Bluetooth names to mobile phones in the UK, and this paper's main contribution is to provide an account of those Bluetooth naming practices, putting them in their social, physical and intentional context. The paper also uncovers how users have appropriated the ways in which Bluetooth, with its relatively short range of about $10-100 \mathrm{~m}$, operates between their mobile phones as a partially embodied medium, making it a distinctive paradigm of socially and physically embedded communication.
\end{abstract}

Keywords: Bluetooth, electronic identity, naming, mobile phones.

\section{Introduction}

This paper reports the results of an in-depth study of Bluetooth naming practices which took place in the UK in August 2006. There is a significant culture of naming phones and other devices in the UK [6], and this paper's contribution is to give an account of those Bluetooth naming practices, putting them in their social, physical and intentional context. This study reveals personal and social naming behaviours that go beyond the initial design of this de facto pervasive technology.

Bluetooth's overall popularity has been increasing markedly since it was integrated in mobile phones, especially in Europe. It was integrated in about half of all phones shipped in Western Europe in 2005; that figure is forecast to rise to about three quarters of mobile phones by 2008 [2]. Much of its use is relatively unremarkable, as a "cable replacement" for connecting phones to other devices such as wireless earpieces. However, a significant use of Bluetooth has emerged as an ad hoc channel for media sharing between users' phones when face-to-face, in places such as schools, pubs and transportation. Indeed, that usage is significant enough to have given rise to recent concerns about Bluetooth-equipped phones as a peer-to-peer channel for bringing pornographic material into schools [1]. This study shows that, in general, the sharing of media has led to an electronic projection of identity and presence through the Bluetooth channel, a presence which runs in parallel to the physical information 
flows within face-to-face engagements. Gary T. Marx writes, in reference to a discussion about privacy and anonymity [5]:

"Some information is always evident in face-to-face interaction because we are all ambulatory autobiographies continuously and unavoidably emitting data for other's senses and machines. The uncontrollable leakage of some information is a condition of physical and social existence."

As this account will show, both controlled (intentional) and uncontrolled projections of our "ambulatory autobiographies" occurs through Bluetooth. Users deliberately project aspects of themselves in their Bluetooth names. At the same time, there is spillage of information that flows via Bluetooth within the immediate social group, and beyond it to or from others in the physical vicinity. Bluetooth, which has a relatively short range of about $10-100 \mathrm{~m}$, is a partially embodied medium. Bluetooth names of mobile phones can't always be matched unambiguously with their owners, who nonetheless are known to be nearby. Bluetooth between mobile phones thus lies somewhere between the wholly embodied medium of a face-to-face conversation, and the wholly disembodied medium of an internet chat between physically dissociated strangers. This paper documents how people make use of and react to the virtualphysical association and disassociation that occurs, as they match (or don't match) information between their sensory channels and their phones' Bluetooth channels.

\section{Related Work}

A previous project also studied how users appropriated an electronic naming feature, this time in the case of instant messaging (IM). Smale and Greenberg [11] captured the usage of IM clients which "include a feature that lets a person create and/or change their display name at any time". The study showed that users appropriated the field designed originally to display their name to other users. While many used it to identify themselves, others used the display field to provide information about themselves, or to broadcast messages, either instead of or in addition to their handle. Subsequent to that study (in 2005), IM clients began providing a dedicated field for a personal message to be shown to other users.

Several Bluetooth-based mobile phone applications have been designed for swapping profile information beyond a simple name, and exchanging messages with others nearby. Examples are Nokia Sensor [7], and MobiLuck (www.mobiluck.com). Even without Bluetooth, the phone is already a way of projecting one's identity. Skog [10] describes how the mobile phone "has become harnessed as part of many a teen's identity project". Similarly, Plant [8] describes its ostensive use as a physical prop to project something about the owner. For example, she describes "stage-phoning", where the phone's owner plays to an audience, for example on a train, in making a call. That language is redolent of Goffman's characterisation of action in social settings as taking place in regions of perceptability [3]: a "front region" where the actor deliberately projects a "performance"; and the "back region", not normally perceived by the same "audience", where that performance is prepared.

It is already established that Bluetooth also allows users to project their identity through their mobile phones, even without special software applications such as 
Mobiluck. At least two projects have shown evidence of Bluetooth naming culture as an apparent performance of sorts. The current authors, in a 2005/2006 study [6], elaborated below, found a very rich set of Bluetooth names when automatically scanning in public places in the city of Bristol, UK. The Mobitip project [9] reported that in 2004 a remarkably high percentage of people's phones were discoverable by Bluetooth in Kista, Sweden, and that some users had changed the default Bluetooth names of their phones to interesting alternatives such as "Keep out!". But neither study established the context behind the names they discovered.

\section{Background}

We begin with an introduction to the essential features of Bluetooth communication needed to understand the findings of this study. Bluetooth, named after a $10^{\text {th }}$-century Viking king credited with unifying Denmark, is a short-range wireless networking technology used for several purposes, including connecting accessories such as earpieces and keyboards to devices such as computers, phones and PDAs; synchronizing mobile devices to computers; and, the use that features most in this paper, transferring content between mobile devices, principally phones. Devices connected by Bluetooth can exchange data at a nominal rate of $1 \mathrm{Mbps}$ in the commonly implemented version 1.2 of the specification. That is enough to transfer media such as a sound file, image or short video clip within a few tens of seconds.

In practice, the devices being connected are typically at most a few metres apart. However, the typical range of a Bluetooth connection is about 10-100 metres, depending on the power of the radio transceivers in the devices and on whether there are obstructions in the environment. Bluetooth radio waves can travel through most obstructions such as walls found in buildings, although they are subject to attenuation. Users cannot control the radio range from their devices.

Often, a user needs to connect his or her device to a target device without the benefit of pre-established connection information. Rather than identifying the target device directly, the user has to select the target from the Bluetooth-enabled devices in range. The electronic process by which a device finds devices available for connection is known as discovery. Bluetooth's range may be relatively short, but many devices may be in range in urban settings such as pubs. We found tens of devices simultaneously present in some crowded places. Discovered devices appear by name, sometimes along with other information such as the type of the device (for example, a phone, a printer or a PC). By default, the name is set to the model of the device, e.g. "Nokia 6680". But the owners of devices such as phones with a means of user input can assign names of their choosing. A device name does not have to be unique. Although names of up to 248 characters are allowed by the specification, we found that user-chosen names are typically short - up to a few tens of characters, which is all that small devices tend to display of a name, anyway.

Discovery can take up to tens of seconds. Once one device has discovered another, a short-cut may be set up between them in the form of a long-term association called a pairing. Thenceforth, each device records the name of the other, together with other information necessary to make the connection. A user may connect the two simply by selecting the other from the pre-established list of paired devices, without the need for discovery. 
Table 1. Names from automatic scans. Ellipses and asterisks replace characters given fully in the original.

\begin{tabular}{|c|c|c|c|}
\hline Identifier & Association & Graffiti/T-Shirt & Direct Address \\
\hline Adam $\mathrm{J} \mathrm{T}^{* * * * * *}$ & The Man From & 4 a gay time call $07 \ldots$ & bonjour tutti! \\
\hline Ami & Delmonte & $\mathrm{B} \tilde{\mathrm{A}} \mathrm{z}$ & Call_me \\
\hline Anna's phone & Biker chic & $>>\} \hat{A} ¥\{<<$ & Clear off! \\
\hline x x carla x x & Bolton & Ho in training & $\mathrm{F}^{* *} \mathrm{k}$ off $\mathrm{u} \mathrm{c}^{* *} \mathrm{t}$ \\
\hline Chris $07 \ldots$ & Everton & Ima kettle & Jokes On U \\
\hline Crown Jools & Pezza's girl & $\$ \mathrm{LiK}$ JiM & Porn please \\
\hline Messy Dawg & Beer boy & MilkWasABad Choice & Tish-Send Me Stuff! \\
\hline Snagglepuss & Madonna & M.C.F.C OK! & VIRUS ALERT!! \\
\hline
\end{tabular}

Finally, devices with Bluetooth enable their owners to control their own radio "presence". The user may switch Bluetooth off entirely while the device still functions. However, there is an intermediate state whereby Bluetooth is switched on, but the device is not discoverable by other devices. In that state, a user can send media to other devices, and other paired devices can send media to the user's device. But the user's device is "invisible" to other, unpaired devices in the radio sphere.

\subsection{Study of Automatically Captured Names}

In a previous study [6], we set up computers to scan continuously for the names of Bluetooth devices in three locations in the city of Bristol, UK: the university campus, a street in the city centre, and inside a pub in the city centre. We collected 1703 Bluetooth names: 771 from the city centre street, 307 from the pub and 625 from the campus. The great majority of these were names of mobile phones. We classified the names according to whether they were default names such as "Nokia 6680", or variations from those defaults that had been set by users. We found that $58 \%$ of discoverable devices had user-defined names in the city centre street; for the campus, the figure was $76 \%$; and for the pub it was $88 \%$.

We found that the vast majority of user-defined names fell into four categories (Table 1), when classified by their apparent meaning. Identifiers include full names, nicknames and pseudonyms. Associations are statements of the owner's interests, such as band names (The Man from Delmonte), football clubs (Bolton), and even a relationship to someone else (Pezza's Girl). Graffiti/T-Shirt includes text liable to be found on a wall, toilet door or a T-shirt. They include decorative forms of names (BÃ@) , jokes and surreality (MilkWasABad Choice) and the scatological (Ho in training). Direct Address includes exclamations that appear to address a person who has discovered the device. Examples include greetings (bonjour tutti!), invitations (Porn please), and insults ( $\mathrm{F}^{* * \mathrm{k}}$ off $\left.\mathrm{u} \mathrm{c} * * \mathrm{t}\right)$.

This a priori categorisation serves to broadly characterise the spread of names we found, and may seem uncontroversial in the case of many of the names we collected. But it was inadequate in several ways. Some of the names were ambiguous. For example, "Everton" is the name of a football club but it is also a boy's name. Some 
names could have been placed in more than one category. For example, "M.C.F.C OK!" refers to Manchester City Football Club but is commonly found as graffiti. But the deeper objection is that, since the exercise was based only on the names' apparent significance, it throws no light on the user's actual intentions or any other aspect of the context within which those names were chosen. What were the Bluetooth names in Table 1 intended to convey? To whom? And in what circumstances?

\section{Methods}

To answer our questions about Bluetooth naming practices and the context in which they take place, we gathered data through semi-structured interviews. Twenty-nine individuals were recruited to the study using a random sampling method and were eligible for inclusion providing they used Bluetooth. Participants were recruited in the daytime outside the Watershed Media Centre in Bristol, which is located adjacent to the recently redeveloped harbourside and includes a café, bar and cinema. It is worth noting however that the broader catchment of the harbourside is not limited to businesses with an interest in media. With local tourist attractions nearby, the area is frequented by a wide range of individuals, which was a major reason for choosing it.

To recruit participants, people walking by the Watershed were simply asked "Have you got Bluetooth?" As this study was not seeking to capture data on why individuals didn't have Bluetooth or didn't use it, individuals in those categories were not invited to participate. Individuals who used Bluetooth were invited to take part in the study through a recorded semi-structured interview conducted inside the Watershed's café. The interviews, which lasted up to about 30 minutes, were given in return for light refreshments and were subject to the interviewee signing a consent form. The two authors of this paper conducted the interviews, together at first so as to agree on the details of the procedure in situ, then separately.

Our introductory question served as a filter prior to the full interview. As no eligibility criteria were included, a wide range of people from different age, sex and ethnicity groups were approached to take part in the study. Age, sex and employment type of participants were recorded, however ethnicity was not recorded. Ten participants were female and 19 were male. The majority of the participants (19) were in the age range 15-25; nine were 26-35 and the remaining participant was 36-45. Twenty participants were in employment at least part-time; seven were students and the other two were unemployed.

The semi-structured interview had previously been piloted with five individuals (whose data are not reported as part of this paper) and minor amendments made. The initial section of the interview contained a series of socio-demographic questions relating to phone model, how often Bluetooth was used, what people did with Bluetooth, whether the participant changed Bluetooth discoverability and whether the participant was able to remember how to change the Bluetooth name of their mobile phone. Interestingly, although the filtering question didn't exclude other devices, all participants discussed Bluetooth use in relation to their mobile phone. Henceforth, Bluetooth is always taken in this paper to be used with mobile phones.

The second section of the interview concentrated on naming practices, including the Bluetooth names participants had selected for their mobile phone, the physical 
context in which the name was set (e.g. home, workplace or elsewhere), the social context (alone, friends, family or work colleagues etc.), the reason behind choosing a particular name and at least one occurrence of something that had happened when that name had been used (e.g. "I sent an image of my dog from my phone to my sister using Bluetooth").

The final section of the interview concentrated on what the participant thought about Bluetooth in general and what, if anything, they would like to change about it. It also captured data on other elements of context or naming practices not captured elsewhere.

Whilst less intrusive questions were asked initially to help guard against selffiltering and response-regulation by participants, it is unknown whether participants were reluctant to report on more provocative names such as some of those previously captured by automatic scanning. This highlights an important methodological consideration, particularly as the names reported in the results section below are not representative of the Bluetooth names in the Direct Address category, observed in the previous study [6].

\section{Results}

Results are presented for the 29 participants who were interviewed for this study and percentages are presented where applicable to highlight the points made. The percentages are presented, however, with the acknowledgement that a relatively small sample was interviewed.

\subsection{Bluetooth and Media Sharing}

Bluetooth was used primarily as a media-sharing tool on mobile phones, between family, friends and work colleagues and, much less frequently, between strangers. We now describe those media-sharing practices because they are the driving force behind the Bluetooth naming practices we shall go on to describe.

Pictures (both those taken with the phone camera and pictures from other sources) and music files are the most exchanged media between participants, with the majority of exchanges taking place between groups in highly social situations such as bars, cafes and at school or the workplace. Other media exchanged included contact information and videos although this was to a much lesser extent. Videos may be exchanged less often due to their size and the relatively slow speed associated with exchanging these files using Bluetooth.

Participants tended to send and receive media on a regular basis with $24 \%$ of participants sending at least one form of media on a daily basis and $10 \%$ of these sending several media files a day. Additionally, $48 \%$ of participants sent media at least once a week and $21 \%$ sent media every 2-3 days suggesting a high rate of media exchange between participants with Bluetooth. Receiving also occurs at a similar rate with $24 \%$ of participants receiving media files on a daily basis and $14 \%$ receiving multiple media files a day. Twenty-eight percent of participants received at least one media file a week and another $28 \%$ received at least one media file a month. 
These figures suggest there is significant social value in media sharing between Bluetooth users. That was confirmed by the comments of some of our study participants. Sometimes the primary value of the exercise was not derived simply from the content, but also from the transaction itself. For instance, one participant described how he would play jokes on his friends by claiming, in one example he gave, to be sending them a "sexy girl" when what he actually sent was a not particularly attractive character from East Enders (a popular BBC "soap"). Another participant described media-sharing as a "good way to socialise", because it gave him and his friends "more to discuss". They listen to music on their friends' phones and then ask them to send the tracks they like. This corresponds to the physical exchange of camera phones for browsing, prior to exchanging selected images [4]. Overall, the combination of mobile phones and Bluetooth makes sharing very convenient, as our study participants commented: the mobility of the devices means that sharing can take place in socially convenient settings where people meet up with their acquaintances, and Bluetooth is free of charge and relatively efficient for exchanging small files.

\subsection{The Bluetooth Names}

Participants were asked to disclose up to three Bluetooth names that they had assigned to their current mobile phone. Only two of the 29 participants had never set their phone's name. Table 2 gives the 33 names we collected, of which six were second or third names from the same participant. The names were largely expressive of a personal or group identity, and indeed a stable identity: only five of the 29 participants had changed the name of their phone more than once, over an average of seven months for which the participants overall had possessed the phone.

The contexts in which participants named their phones confirmed there was an established awareness of Bluetooth in many cases. The initial naming of the mobile phones occurs largely when setting up the phone for the first time or browsing the features on the phone during an idle moment. About $70 \%$ of participants named their phone in those circumstances. The remaining $30 \%$ of participants named the phone when they were using Bluetooth to transfer files. Initial naming tends to occur when the participant is at home. Just over half of participants named their phone when at home; other places included work, during journeys, when out and about on the street, or in a venue such as a pub or fast-food restaurant. Overall, 57\% of participants were with someone else (family member, friend or colleague) when they named the phone.

The contexts in which Bluetooth names were commonly set also suggest that naming the phone is an aspect of personalizing it in some general sense, and several participants described it in those terms. Equally, however, there was awareness that naming the phone had a practical purpose: to eliminate ambiguity when sharing media. In settings such as pubs, tens of phones may be simultaneously discoverable. There may, for example, be two phones with a default name such as "Nokia 6680". There is no way of distinguishing the phones without attempting to transfer media to one of them, which users are understandably reluctant to attempt. Participants who share media in public places therefore tend to re-name their device from the default to a less ambiguous name. Importantly, as will be shown, that new name is one which participants in their immediate social groups will recognise or respond to 
Table 2. Participant names. Superscripts denote names of same participant. Asterisks replace letters given fully in the original. Names are in lower case irrespective of the original.

\begin{tabular}{|c|c|c|c|c|c|}
\hline $\begin{array}{l}\text { Name of } \\
\text { my phone }\end{array}$ & \multicolumn{2}{|c|}{$\begin{array}{l}\text { Name for me } \\
\text { (includes nickname or alias) }\end{array}$} & Statement about me & $\begin{array}{l}\text { More like } \\
\text { graffiti }\end{array}$ & Other \\
\hline \multirow{9}{*}{$\begin{array}{l}\text { sami's } \\
\text { phone }\end{array}$} & zoe & amon & caged_gardener ${ }^{4}$ & lilla & nk83 \\
\hline & adamcall & popeye & $\begin{array}{l}\text { caged_gardener@** } \\
* . \text { co.uk }^{4}\end{array}$ & \multirow[t]{8}{*}{ soop } & $\begin{array}{l}\text { get your } \\
\text { em out }\end{array}$ \\
\hline & shell & smokey $^{2}$ & luddite $^{4}$ & & $\begin{array}{l}\text { merolyn } \\
\text { the phone }\end{array}$ \\
\hline & jasey & nokia ham & almost dr dee & & leroy $^{3}$ \\
\hline & $\begin{array}{l}\text { ben's } \\
\text { mobile }\end{array}$ & nkwile & the mac man & & boop $^{1}$ \\
\hline & james & star & blue army ${ }^{5}$ & & \multirow[t]{4}{*}{ raspclart } \\
\hline & $\mathrm{klc}^{1}$ & winston $^{3}$ & b.a. baracus ${ }^{5}$ & & \\
\hline & ryan $^{2}$ & lady hype & optimus prime $^{5}$ & & \\
\hline & & paulw810i & fuc***g $\mathrm{bi}^{* * \mathrm{~h}}$ & & \\
\hline
\end{tabular}

Turning to the names themselves (Table 2), in order to understand the intention behind the choice of each name, participants were asked both why they had chosen that particular name and what type of name it was. On the basis of the prior study of automatically scanned names, participants were asked whether their name's type was one of the categories "Name of my phone", "Name for me", "Statement about me" or "More like graffiti". Alternatively, they could state that it was of an "other" type. Only a fifth (seven) of the total of 35 names belonged to the "other" category.

The answers to the questions "Why that particular name?" and "What type of name is this?" gave a consistent picture of the intentions behind each choice of name which emerges as we consider the names of each type.

Name of my phone. Only one name, "sami's phone", was declared to be the name of the device itself; this participant expressed a functional view of the names he used. All but one of the other names ("nk83", discussed later) were connected with the identity of the owner in some way.

Name for me. The largest category, at just under half of the Bluetooth names, was that of a name of the phone's owner - including nicknames or other aliases. Some of these were part of the participants' bone fide names. A few first names appeared (zoe, ryan, james). The name "klc" is the owner's initials. The closest name to a full name is "adamcall", whose last four letters are the first of the participant's surname. Participants "klc" and "adamcall" were concerned to make their Bluetooth names unique but identifiable to their social groups, without fully identifying themselves to others. A similar motivation lay behind some hybrid names that also contained a reference to the device itself. Note that the owner of "ben's mobile" classified it as his own name, even though it is of the same form as "sami's phone"; similarly, "paulw810i", which contains the phone model, was in this category.

This category also includes participants' regular nicknames as used by their friends. As such, they have a variety of origins. For example, the names "amon" and 
"shell" are contractions of first names, and "ham" in "nokia ham" refers to the participant's last name, used as a nickname. The nickname "smokey" was adopted by a participant who is a runner and the name is in reference to his running pace.

Adoption of first names and nicknames as Bluetooth names enables familiars to recognise the name when sharing media, albeit with some possibility of ambiguity. An interesting special case is "jasey". His name is Jason but he also had a friend by the same name. To prevent ambiguity when receiving media from friends, he decided to adopt the name "jasey", although this was not a previously used nickname.

There were also a few cases where participants used an alias that was not a nickname used regularly by their acquaintances, and rather was more an expression of some aspect of their wider identity that they wanted to bring into the Bluetooth realm. Indeed, these participants' interview comments suggest that a classification under our category "statement about me" might also have been appropriate, despite the fact that they chose our category "name for me". "Lady hype" uses that name as a (hip-hop) MC name. "Nkwile" used that name as a contraction of the name "Wile-Kat" which he adopts widely in internet chat rooms ("nk" refers to his Nokia phone). A quite different example of an alias is "Winston". This Bluetooth name belongs to a black participant who chose the name as it reflected a stereotypical afro-carribean name. His explanation was that "it's kind of a mickey-take out of myself".

Statement about me. Names in this category express some aspects of the phone's owner. "Caged_gardener" chose a name that was reflective of his personal situation he is a professional gardener living in the suburbs of Bristol and feels constrained by the suburban setting where he lives. That participant previously used his entire email address “caged_gardener@***.co.uk" as his Bluetooth name - as an invitation for others to contact him. This participant generally chose names to differentiate himself and gain a reaction from others; he formerly used "luddite", referring to his innate skepticism about technology, in order to "express an attitude that differentiated me from most people I know". "Almost dr dee" is a PhD student who is proud of his ambition to graduate with a doctorate. "The mac man" wanted playfully to distinguish himself from his colleagues, who use Windows PCs. The name "blue army" belongs to an Ipswich Town football fan. His other names "b.a. baracus" and "optimus prime" stem from his liking for 1980s TV series. Finally, "fuc***g bi**h" (without asterisks on her phone), adopted that name because "I think that's what I am".

More like Graffiti. The participant "soop" made up the name solely for his Bluetooth phone. He described that name as a kind of "handle", a term sometimes used as a synonym for "tag" in graffiti. The Bluetooth name "lilla" belongs to a participant who is in fact a graffiti artist and tags objects (buildings, walls, train carriages) using the name "Lilla".

Other. This category contains examples that are quite distinct from names in the other categories. First, the Bluetooth name "get your em out" is a unique case exemplifying the coupling of both social in-grouping and the inclusion of a participant's own identity. This participant chose a name of the form "get your out" as it is a phrase used amongst her friendship group, but decided to include her name in place of any other more arbitrary object. Choosing such a Bluetooth name reinforces the 
participant's position of belonging to this particular friendship group (since this is an "in" phrase within the group), and in turn enables easy discovery by others in the group who may want to share media with the participant - since her real name "Em" is also included within the Bluetooth name. Another example of a group-specific choice of name was "Raspclart", which was offered spontaneously as a "funny" name by a friend of the participant - and where choosing "funny" names was part of the culture of the group.

In the rest of the "other" category, the participants used Bluetooth names not to express something about their true identity or that of their group, but for some other purpose. Two names are examples of concealment involving someone else's name in a Bluetooth name. "Leroy" is the real-world name of a friend of the person otherwise known in the Bluetooth realm as "winston". "Winston"/"leroy" actually changes his Bluetooth name according to the social context, and, in particular, according to which girlfriend he is with! A different participant also changed his Bluetooth name because of a girlfriend, but he did so long-term. This is the case of "merolyn the phone", where "merolyn" was the name of an ex-girlfriend that the participant spent considerable time with. Initially the Bluetooth name was simply "merolyn" but now the participant has a new girlfriend (who isn't called Merolyn), the participant changed the name to include the phrase "the phone", in order to remove the direct association with another girl's name.

The remaining names that participants placed in the "other" category were chosen spontaneously as distinctive identifiers, using whatever basis was conveniently to hand, but with quite different intentions. One participant chose "boop" because she wanted to hide her usual Bluetooth identity by way of a riddle to her friends, and had looked at Betty Boop merchandise in a shop that day. The final participant had a purely functional goal of unique identification in mind: he synthesised "nk83" as a hybrid "identifier" (his term) from the phone's manufacturer in the default name, Nokia, and his year of birth (83).

Turning to the collection of names as a whole, several themes emerge. First, the participants were largely naming their phones for the benefit of those they knew or directly encountered, not for strangers. It is unknown whether names such as "send me porn" in the Direct Address category of our previous study were addressed to strangers. (They might have been for the amusement of friends.) But, as we shall see, only two participants, "caged_gardener@***.co.uk" and "lilla", intended through their choice of name to obtain a reaction from strangers (by electronic means).

The second, overlapping, theme is that the social sphere constituted by the group and beyond often impinges on the choice of names. This is clearly true in respect of the participants' use of their own everyday names and nicknames. Indeed, when asked why they had chosen those names as their Bluetooth names, many responded "Because it's my (nick)name!" - as though it was obvious to transcribe it into the Bluetooth realm, and no further explanation was required. But some were names from somewhat more esoteric aspects of the participants' lives ("lilla" as a graffiti tag, "lady hype" as an MC name), and names used in the virtual world of electronic communication. "Lilla" was also used in online social communities (Microsoft Messenger and MySpace); "nkwile" derives from a chat name; and "caged_gardener" was first used in an email context. 
In the latter cases, the participant specifically wanted to draw attention to his or her activities outside the use of Bluetooth. "Lilla" explained that the reason the same tagging name was used in a virtual world was to see if people would recognise this name and attempt to make contact, identifying the participant as an acknowledged graffiti artist in the physical world. This demonstrates how a participant was using a Bluetooth name to attract strangers and encourage communication and/or media sharing, rather than the participant declaring this aspect of his or her identity to others. "caged_gardener@***.co.uk" was curious whether anyone would contact him - and he reported that indeed someone emailed him to explain that he or she had encountered the address while scanning for Bluetooth devices. "Lady hype" wanted people around her to discuss her MC activities with her.

The desire for interaction in the foregoing examples is reflected throughout many of the remaining participants, especially those with Bluetooth names in the "statement about me" category. Participants in that category reported that they want their names to be noticed and to become a topic of conversation - for example, "almost dr dee" wanted "everyone to know" about his "ambition". As the examples show, Bluetooth enables people to express aspects of their identity in a relatively self-effacing way.

Finally, there is the observation that participants changed their Bluetooth names very little overall. Of the 27 participants in total who set the name at all on their current phone, only five did so more than once. That is arguably due to the reasons most had for choosing their names - such as making their phones easily identifiable as targets for media files - which themselves are quite stably connected with the participant's identity. Only one, "winston"/"leroy", changed his name regularly according to the social context - on the basis of which city he was in and which girlfriend he was with. His choice of Bluetooth names was somewhat ambiguous, being both playful and light-hearted on the one hand and used with more serious intent on the other. The other cases of name-changing are more definitely playful. "Klc" changed her Bluetooth name to "boop" during a football match when she was bored and wanted to send a file to her friends from an unknown source, to see their reactions. "Caged_gardner" changed his name as a result of projecting different aspects of his personality (he was previously "luddite"), and during a temporary experiment with his entire email address. And the participant known as "blue army" / "b.a. baracus" / "optimus prime" was playfully projecting whatever specific interest caught his imagination from time to time.

\subsection{In-Group Conversations About Bluetooth Names}

Just as the participants projected their own identity through their choice of Bluetooth names, they also showed interest in the Bluetooth-projected identities of others in their social groups. In addition to asking participants about their own Bluetooth names, we also asked about the Bluetooth names of their friends, family and colleagues. The majority of those interviewed were able to report at least one Bluetooth name of someone they knew from memory, and often several. The names that others had chosen were usually learned when sharing media. On occasion, learning someone's Bluetooth name resulted in further discussion and exploration of that name. Several examples appear above of participants choosing names that invite interaction from strangers; the same applies within their social groups. 
Sometimes, to learn a Bluetooth name is to learn something surprising about the owner. "Almost dr dee" for example commented on his surprise when learning the Bluetooth name "mrs prince william" of his friend. He was surprised that she had this "fantasy" and he had not previously known anything about it. Similarly, "lilla" asked a friend about his Bluetooth name ("crisis") as he thought the name was "strange". His friend, who was a DJ, explained that when in Bristol he uses his real name but changes his name when in London to "crisis" as this is the DJ name he uses there. This is another example of name-changing in context with the social situation. "Fuc***g bi**h" was surprised and confused as to why someone she had known for a long time chose the name "rocking horse $\mathrm{s}^{* * \mathrm{t}}$ ". When questioned the friend explained that the name referred to the saying "as rare as rocking horse $\mathrm{s} * * \mathrm{t}$ ", and as he liked the phrase he decided to adopt this as Bluetooth name. She first discovered his name when he was trying to send her an image from his mobile phone, supporting the notion that discussion of names occurs when media sharing is the primary activity.

Other participants also expressed surprise at the Bluetooth names of their friends and work colleagues, and sought to understand the reasoning behind the choice of name, suggesting that the culture of naming Bluetooth devices does lead to intrigue and occasional ambiguity even between familiar individuals. By the nature of Bluetooth discovery, sharing media with one individual usually entails becoming aware of other names around - names whose owner might not be known. For example, "amon" discovered the unfamiliar name "spongemonkey" when at the hotel where she works. The name baffled her but she felt able to ask the group of colleagues around her. A chef nearby responded that he used this Bluetooth name to reflect the fact that he makes the cakes.

As reported above, an interesting case where one participant deliberately created a conversation in her group about a Bluetooth name was "boop". She set her Bluetooth name to "boop" to hide her identity when sending files to her friends at a football match as a joke. She had the satisfaction of waiting until her friends had wondered who the sender was, before revealing herself.

Not all the interaction centred around Bluetooth name discovery was verbal. "The mac man" reports that because he is an Apple Macintosh user and has adopted this as part of his Bluetooth name, his friends have seen this as an opportunity to reflect the real-word rivalry between the two companies by sending him pictures of the Windows logo.

\subsection{Interest in Bluetooth Names Beyond the Social Group}

We have described the interest that some participants found in others' names within their social groups, and interactions that followed the discovery of those names. But some participants also showed interest in the names of strangers around them, and even interacted with them.

Participants were asked whether they ever browsed for Bluetooth names, and why they did so and in what circumstances. By "browsing" we mean the activity of a user setting his or her phone to discover all the Bluetooth names in range for the sake of doing so, rather than in the context of a specific goal such as media sharing. Almost three quarters of the 29 participants reported they sometimes browsed for Bluetooth names. Thirteen participants said that they did so about once a month or more, and 


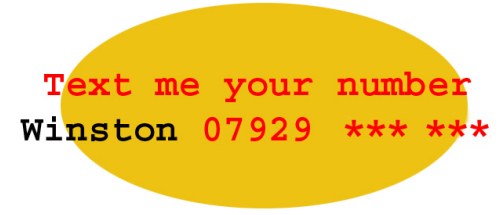

Fig. 1. Winston's image. Asterisks replace digits given fully in the original seven browsed at least once a week. Browsing typically took place in leisure settings and places of dwelling, including pubs, concerts, trains and buses. Most said that they browsed only when bored, and that they did so for casual reasons including "out of curiosity", "to see how many people have it turned on" and "to see what people call themselves". Objects of

particular interest in these cases were typically names that were, as two participants put it, "funny" or "strange".

Two participants had more focused interests when browsing. "Lilla", the tagger, looked for others who used their tags as Bluetooth names. "Popeye", who browsed at work during his lunch hour, was interested in the models of other people's phones: to "see what phones were out there" - he was not interested in the unusual names people had set for themselves.

\subsection{Spillage}

To assign a name and make a Bluetooth device discoverable is to project an electronic presence, but not all aspects of Bluetooth presence are under the user's control. This section describes ways in which Bluetooth presences "spill" both between social groups and between the virtual and physical worlds.

The people browsing for Bluetooth names in the previous section specifically looked for the names of strangers. But eight of the 29 participants used discovery only to find the device of an acquaintance, and were uninterested in the other names they observed. However, they had no choice in the matter: the presences of strangers "spill" into the discovery process even though they are looking for those of people they know. Given the public or semi-public contexts in which Bluetooth is typically used, the appearances of presences from beyond the immediate, known social group is not within the user's control. In most cases, this spillage of Bluetooth presences was of little importance. This type of spillage may be termed "overdiscovery", by analogy with overhearing - which is usually of little consequence. But two of those eight participants had concerns about it. One man said that he wanted to discover only friends' phones "because of security", although he wasn't able to elaborate on what he meant by that. The other said she was "shocked" at some of the "rude stuff" she saw: "what kind of person would call themselves that?" Her particular concern was that that "kind of person" must be nearby.

Her concern arises from a relationship between virtual Bluetooth presence and physical presence. Because Bluetooth is a short-range medium, there is a degree of correlation of this electronic presence with the device owner's physical presence: as the introduction stated, Bluetooth is in that sense a partially embodied form of communication. There are several ways in which these two forms of presence "spill" over into one another. In that respect, Bluetooth presence bears some similarity to WiFi presence. For example, an unfamiliar network observed when a user connects a laptop within the home must be nearby. Even on a wired network, there are presences 
of users known to be physically nearby. For example, the play-list discovered when running the iTunes application exists on the same subnet, and therefore belongs to someone nearby. But in both those cases, the environments in which the user discovers the other presence are well-known and relatively slow-changing - they are observed as belonging to our neighbours, colleagues or fellow students. As Bluetooth users move around urban places, they typically know less about the changing presences nearby.

Bluetooth presence is also comparable to online presence, such as in instantmessaging and chat rooms, which is normally wholly disembodied. In one respect, Bluetooth presence is more anonymous: beyond the Bluetooth name, there is no further information such as a picture or profile to identify the person. On the other hand, while a user may remain anonymous despite Bluetooth presence, the existence of correlated physical information channels may creates ways to break anonymity.

Some participants tried to varying extents to physically identify a person from his or her Bluetooth name. "Caged_gardener" tried to "match faces to names" - an act that went no further than his imagination. But three participants "Bluejacked" others: that is, having discovered their names, they sent them unsolicited content by Bluetooth. In each case, the goal was more than sending content per se: it was to have an effect on a recognizable person. One of these, "soop", sent a message to a stranger's device containing the text "phone will shut down". He did so using common Bluejacking practice, as a spurious "contact" message, which some phones accept automatically. Then he looked around to see who had received it. A person who receives such a message is liable to pick up his or her phone, and examine the contents. When it contains a phrase such as "phone will shut down", the recipient is liable to show concern or amusement, and to look around to see whether they can spot who sent it. In such circumstances, the sender may be able to identify the target with reasonable probability.

That identification doesn't necessarily work both ways. Another participant, "blue army", sent an image of Mr. T. (from "The A Team", a 1980s TV program) to a female-sounding name in a pub. He saw a girl pick up her phone. She sent back an image of a rock band, apparently without being able to see him - his intention was not to be identified himself.

The third example, of "Winston", was particularly interesting because his intention was to be identified, although he went about things in a way that stood little chance of success. He wanted to give his telephone number to women he felt attracted to. $\mathrm{He}$ constructed the image shown in Figure 1 with his telephone number for that purpose. On seeing a woman he liked, he would try to discover a Bluetooth name that he thought might be hers, and his plan was to send the image to her. But he never succeeded in discovering a name that he considered might belong to a woman he had spotted. If he had succeeded, he planned to make himself known when he observed the target receiving the image. He viewed Bluetooth as a "cool" way to introduce or project himself.

Some participants described spillage occurring the other way: that is, of others' attempts to contact them. "B.a.baracus" (also known as "blue army") once received an unsolicited image in a bar. He does not know whether the perpetrator identified him, but it gave him the idea of trying the same on someone else, as reported above. "Ryan" was identified in a very crude way: on a bus, a young woman he didn't know 
shouted "who's Ryan?", during a bout of Bluetooth activity between young people. He reports that he identified himself to the girl who had asked, even though he thought it was "pointless" of her. However, he remarked that he chose the name "smokey" not only because it was his nickname, but that, he felt, that "it's not really a name" and would "get fewer comments".

Sometimes the physical domain spills into the world of Bluetooth presences, as examples brought up by "amon" show. The first example is of the physical resolution of a problem with a Bluetooth transaction. She reports a situation when travelling on a bus of an unsolicited image being sent to her via Bluetooth. When she expressed surprise at receiving the image, the sender (also on the bus) overheard and apologized to her, stating that the file was intended for someone with the Bluetooth name "Amon3000". In the second example, the same participant answered a question from one of her colleagues, who did not know her personally, about her Bluetooth name. She wore a T-shirt bearing her nickname, Amon, and the colleague explained to her that he had previously known her only as a disembodied Bluetooth name.

\subsection{Controlling Bluetooth Presence}

Technically, Bluetooth can either be switched off entirely, or left on with discoverability turned off. The participants tended to be unclear about the difference.

Ten of the 29 participants said that they left Bluetooth on all the time; the remainder turned it "off" sometimes. Of those 19 who sometimes turned it off, 15 did so because of security considerations or because of Bluetooth's drain on battery life (or both), and were evenly divided between those issues; the remaining four gave other reasons or no definite response. Those concerned about battery life switched Bluetooth off when it was not needed, or when they noticed the battery getting low.

Of more interest here are the nine who mentioned security considerations. Their issues ranged from irritations such as erroneous headset connection attempts, to fear of "hacking". Four participants mentioned particular places where they turned Bluetooth off: at one participant's place of work (where viruses were quite common due to a high density of phones); at McDonald's restaurants, where "people always try and connect"; in "big crowds", where people might be able to "find who you are or get through to you"; and "in Birmingham" - the large city in the English Midlands, where the participant was generally suspicious of the crowds found there!

\section{Discussion}

This study has demonstrated (1) the significant culture around Bluetooth naming of mobile phones, which has emerged from what was designed simply as a mechanism for device association; and (2) the ways in which Bluetooth on mobile phones is a partially embodied medium, and how that plays into its use. Taking the culture first, a small but significant minority of people participate (about $10 \%$ of all people walking along a Bath street over various times had discoverable user-set names [6]), and we have shown that name-setting is rooted in a significant practice of media sharing: about half of our study participants (randomly chosen Bluetooth users) sent media 
files at least once a week. Media-sharing is a valuable social transaction in itself, but the names provide an additional channel for projecting facets of personal identity.

Our interview study provided an explanation for the names in most of our initial lexical categories from the automatic scans, which are now distinguishable by the intentions behind them. Those intentions go beyond the simple need to disambiguate. One is the in-group intention. Many are focused on their immediate social group. Nicknames are a popular choice, because they identify the user in such a way as to emphasise their belonging to the group. Nicknames do so without fully identifying them to anyone beyond, and so tend to maintain a degree of privacy. Some examples of in-group naming went a step further: "get your em out" involves an in-group phrase, and "raspclaart" shares an in-joke. We also found examples of Statement about me names that mirrored our former "association" category, but which we can now distinguish by intention. Those users tend to choose their Bluetooth names to prompt others to ask about them. Sometimes the intention is to find commonality: to directly find people with common interests, e.g. "blue army's" reference to the football team he supports. But sometimes people use the Bluetooth name with an ask me intention: as a prompt for others to find out more about them. They choose their name slightly cryptically ("almost dr dee", "caged_gardener") or even provocatively ("fuc***g bi**h"). A special case of that is the alter ego intention: users such as "lady hype" and "lilla" were drawing attention to identities they possess beyond their immediate social group, as a hip-hop MC and graffiti artist respectively. Finally, we found the obfuscation intention: in contrast to the preceding examples, the users with names "leroy" and "merolyn the phone" were using their names to obfuscate, respectively, a true identity and the significance of an ex-girlfriend's name. Similarly, "boop" was a deceptive Bluetooth identity chosen for a prank.

Some of the lexical classes of names from our initial scans are not represented in the interview study. Those include the Direct Address names such as "Porn please", and the more surreal or scatological Graffiti/T-shirt examples. That may be because the participants were reluctant to tell us about them. As a consequence, we have not been able to answer one of our original questions: what were the intentions behind those most provocative of names, and at whom were they directed? Both in-group amusement and out-of-group affect are plausible rationales. "Raspclaart" is an existence proof of the former. "Lilla" and "caged_gardener@***.co.uk" both sought interactions from people outside their immediate social group.

We now turn to what is perhaps the central question of this study: are Bluetooth names different from other types of electronic names? At first glance, the answer would seem to be 'no'. Many Bluetooth names are similar to those found online. Indeed "lilla" and "caged_gardener" were used online. As for the others, real-life names and nicknames found in the Bluetooth realm are not uncommon in instant messaging, where people message only their "buddies". Some Bluetooth pseudonyms, Statement about me names and graffiti-related names such as "lady hype" and "BÃ@z" seem redolent of online names in broader forums. Even the more scatological Bluetooth names might be found there. The only obvious difference from most online environments is that some Bluetooth names, such as "ben's mobile", mention phones.

However, we argue that Bluetooth naming is a distinct paradigm from other types of personal and electronic naming, not so much in the names themselves, as in the modes in which those names are used - particularly, the modes by which they are 
discovered and disclosed. That position is based on the other major finding of this study: that Bluetooth between mobile phones is what we have termed a partially embodied medium. At one extreme it is virtual or disembodied: one can communicate with a stranger who remains unknown. At the other extreme it is wholly embodied, in situations where the denotation of a Bluetooth name is unambiguous. For example, the "smokey" you have discovered is the Smokey you know at the same table, who is talking about the video you just sent to him. But there are important penumbral cases in which Bluetooth emanates from an unknown person who is known to be physically close, and may become known. That is often a cause of interest or even concern.

Indeed, we have shown that much may hinge on the discovery (or disclosure) that this Bluetooth name belongs to that person, in a way that is substantively different from other realms of interaction. Unlike the online realm, such a discovery is quite probable in many circumstances, and has physical immediacy. Unlike subnetrestricted broadcast of iTunes music, where the owners of network share names also may or may not become known, people carry their Bluetooth names into a variety of circumstances with new opportunities for discovery or disclosure. Unlike ordinary personal names learned in conversation, Bluetooth names are just latent enough to be put to interesting uses. People such as "almost dr dee" even base certain disclosures about themselves on that point of discovery. "Winston" wanted to impress women by discovering them. Moreover, some people play with the difficulty of making the association. "Boop" did that when she sent a file to her friends under an unknown name. "Blue army" could tell he had guessed correctly the Bluetooth name of a girl he saw in a pub, because she picked up her phone when he sent her an image. But she did not know who had sent it. If the pub was a stage, to follow Goffman [3], then this was dramatic irony. In general, its propensity for virtual-physical dissociation followed by a surprising or unwitting association make Bluetooth, used in this way between mobile phones, an ironic medium - a term chosen deliberately to emphasise how humans have appropriated the technology.

\section{Conclusions}

We have shown that Bluetooth between mobile phones represents a distinctive, partially embodied paradigm of identity projection in pervasive communication. It is integrated not simply into the physical world, but into the social world, in a sometimes ambiguous way; and that ambiguity itself plays into social practices.

There is further research to be carried out into the context behind some of the more provocative names found in our initial scans. In the meantime, however, what we have uncovered is not "how to design a better Bluetooth". Indeed, the only such implications the interviews clearly revealed was that users would, of course, prefer more bandwidth and lower battery drain, and some would prefer better security guarantees; otherwise, they were content with Bluetooth as it stands. Rather, this paper has provided a case study in how users have appropriated a pervasive system design. This is somewhat similar to the appropriation of SMS messages, also an exercise in squeezing significance into a small amount of text. However, this study shows how humans deal with an important difference: the partially embodied, relatively uncontrolled ways in which Bluetooth presence flows or 'spills' between 
people. In ongoing research, with a view to generating discussion about spillage and partial embodiment, we are constructing an application that will reveal scanned Bluetooth names on public displays, along with related scanned information about people's social networks and the locations where they were scanned.

Acknowledgments. The authors would like to thank Eamonn O'Neill, Vassilis Kostakos, the anonymous reviewers, and the Watershed Media Centre. This research is funded by the UK Engineering and Physical Sciences Research Council grant EP/C547683/1 (Cityware: urban design and pervasive systems).

\section{References}

1. Bell, R.: Love in the time of phone porn. The Guardian (January 30, 2007) http://education.guardian.co.uk/egweekly/story/0,2001171,00.html

2. Blaber, G., Brown, A., Bouchard, J.-P.: IDC Western European Mobile Phone Forecast and Analysis, 2005-2010 (2006)

3. Goffman, E.: The presentation of self in everyday life, Penguin (1959)

4. Kindberg, T., Spasojevic, M., Sellen, A., Fleck, R.: The ubiquitous camera: an in-depth study of camera phone use. IEEE Pervasive Computing 4(2), 42-50 (2005)

5. Marx, G.T.: What's in a Name? Some Reflections on the Sociology of Anonymity. The Information Society 15(2), 99-112 (1999)

6. O’Neill, E., Kostakos, V., Kindberg, T., gen, F., Schiek, A., Penn, A., Stanton Fraser, D., Jones, T.: Instrumenting the city: developing methods for observing and understanding the digital cityscape. In: Dourish, P., Friday, A. (eds.) UbiComp 2006. LNCS, vol. 4206, Springer, Heidelberg (2006)

7. Persson, P., Jung, Y.: Nokia Sensor: From Research to Product. In: proceedings Conference on Designing for User eXperience (DUX) (2005)

8. Plant, S.: On the Mobile: the effects of mobile telephones on social and individual life. Report commissioned by Motorola (2002)

9. Rudström, Å., Höök, K., Svensson, M.: Social positioning: Designing the Seams between Social, Physical and Digital Space. In: 1st International Conference on Online Communities and Social Computing, at HCII 2005, 24-27 July 2005, Las Vegas, USA (2005)

10. Skog, B.: Mobiles and the Norwegian teen: identity, gender and class. In: Katz, J.E., Aakhus, M. (eds.) Perpetual contact: mobile communication, private talk, public performance, pp. 255-273. Cambridge University Press, New York (2002)

11. Smale, S., Greenberg, S.: Broadcasting information via display names in instant messaging. In: Proceedings of the 2005 international ACM SIGGROUP conference on Supporting group work, pp. 89-98. ACM Press, New York (2005) 